Revue internationale P.M.E.

Économie et gestion de la petite et moyenne entreprise

Revure

internationale

PME

\title{
La continuité dans la PME familiale
}

\section{Yvon Gasse, Ghislain Théberge et Julien Naud}

Volume 1, numéro 1, 1988

URI : https://id.erudit.org/iderudit/1007873ar

DOI : https://doi.org/10.7202/1007873ar

Aller au sommaire du numéro

Éditeur(s)

Presses de l’Université du Québec

ISSN

0776-5436 (imprimé)

1918-9699 (numérique)

Découvrir la revue

Citer cet article

Gasse, Y., Théberge, G. \& Naud, J. (1988). La continuité dans la PME familiale. Revue internationale P.M.E., 1(1), 43-56. https://doi.org/10.7202/1007873ar

\section{Résumé de l'article}

La problématique de la relève - succession au niveau des petites et moyennes entreprises (PME) familiales du Québec, est le fondement majeur de cette étude exploratoire. Celle-ci repose, dans un premier temps, sur la perception qu'ont les propriétaires-dirigeants québécois face à la relève et à la succession; en second lieu, l'étude permet de dégager certaines pistes dans le but de guider les principaux mandataires au niveau des actions à prendre dans ce dossier. Les résultats révèlent que la perception des propriétaires-dirigeants varie selon les secteurs économiques, les formes juridiques, l'âge et la taille de l'entreprise, l'âge du dirigeant, la composition de la famille et l'intégration de ses membres. 


\title{
La continuité dans la PME familiale*
}

\author{
Yvon GASSE \\ Directeur du programme PME \\ Université Laval \\ Ghislain THEBERGE \\ Professeur invité \\ Faculté des sciences sociales \\ Université Laval \\ Julien NAUD \\ Etudiant \\ Faculté des sciences sociales \\ Université Laval
}

\section{RESUME}

La problématique de la relève - succession au niveau des petites et moyennes entreprises (PME) familiales du Québec, est le fondement majeur de cette étude exploratoire. Celle-ci repose, dans un premier temps, sur la perception qu'ont les propriétaires-dirigeants québécois face à la relève et à la succession; en second lieu, l'étude permet de dégager certaines pistes dans le but de guider les principaux mandataires au niveau des actions à prendre dans ce dossier. Les résultats révèlent que la perception des propriétaires-dirigeants varie selon les secteurs économiques, les formes juridiques, l'âge et la taille de l'entreprise, l'âge du dirigeant, la composition de la famille et l'intégration de ses membres.

\section{ABSTRACT}

This study is mainly based on the problem of take over and succession represent from small and medium-sized family businesses in Quebec. First, it tries to find out how owner-managers in Quebec consider take over and succession. Second, the study helps to define proceedings in order to guide the major persons concerned. It analyzes take over and succession within the close family (parents/children). The results show that the concern of owner-managers toward the problem varies with the type of business, the legal proceedings, the age and size of the business, the age of the manager, the structure of the family, and the involvement of its members.

\section{RESUMEN}

La problematica del relevo y de la sucesion a nivel de la pequena y de la mediana empresa (P.M.E.) familiar del Quebec es la base principal de este estudio exploratorio. Este trata primeramente de la percepcion que tienen los propietarios-dirigentes quebecuenses del relevo y de la sucesion. En segundo lugar, el estudio permite desprender algunas pistas que pueden servir de quia a los principales mandatarios, en la toma de acciones pertinentes al caso. Los resultados revelan que la percepcion de los propietarios-dirigentes varia segun los sectores economicos, las formas juridicas, el tamano y el tiempo que lieva la empresa, la edad del dirigente, la composicion de la familia y la integracion de sus miembros.

* Etude supportée financièrement par la direction de l'entrepreneuriat féminin du ministère de l'Industrie et du Commerce du Québec et de l'Institut de Recherches Politiques du Canada. 
La continuité dans la PME familiale

\section{Problématique}

Au cours des dernières années, plusieurs études portant sur l'entrepreneuriat, la PME et, en particulier, sur les propriétaires-dirigeants d'entreprises familiales ont souligné le peu de préoccupations de ces derniers pour les questions de succession et la relève. Par exemple, une étude effectuée par un des auteurs (Gasse, 1977) auprès d'une cinquantaine de propriétaires-dirigeants d'entreprises manufacturières du Québec révélait que la moitié d'entre eux n'avaient pas la moindre idée d'une période, même très approximative, pour prendre leur retraite; de plus, environ le quart de ces dirigeants prévoyaient ne jamais se retirer de leur entreprise.

On peut assez facilement comprendre les sentiments d'un propriétaire d'entreprise qui y a consacré toute sa vie, de ne pas se sentir disposé à parler de retraite; mais tous admettront volontiers l'importance de la relève, surtout au fur et à mesure que l'âge nous rappelle nos limites.

L'étude, dont nous présentons ici une synthèse, se veut surtout un moyen de sensibilisation sur ce sujet, souvent tabou, qu'est la relève managériale et la succession dans la propriété de l'entreprise.

D'entrée de jeu, on peut immédiatement se demander si la succession et la relève dans l'entreprise familiale québécoise constituent de véritables problèmes. Il semble cependant que les gens d'affaires, tout en admettant que ces questions soient importantes n'agissent pas toujours en conséquence. Les résultats de la présente étude sont passablement éloquents à cet égard. Il y a certes beaucoup à améliorer du côté des attitudes, mentalités et comportements des dirigeants et dirigeantes d'entreprises et ce texte fournit quelques pistes à explorer à cet effet mais il ne faut pas pour autant oublier qu'il s'agit d'un processus à deux directions, les successeurs éventuels et potentiels doivent aussi être dans le coup.

On doit l'admettre, le processus de succession est complexe et peut faire intervenir plusieurs parties aux intérêts diversifiés et aux attentes divergentes. Par ailleurs, dans ce domaine comme dans d'autres, une bonne connaissance du sujet et une préparation à long terme peuvent grandement faciliter le déroulement des étapes cruciales. Mais quelles sont-elles ces étapes?

Une étude en cours(Hugron, 1985) parrainée par le Programme de recherches sur la PME de l'Institut de Recherches Politiques nous renseigne sur le sujet. Pour bien comprendre la dynamique de la succession, le professeur Pierre Hugron des HEC, Montréal, a étudié douze cas dans des entreprises familiales au Québec. Il a ainsi été amené à faire une distinction entre la succession managériale (succes-sion à la direction de l'entreprise) et la succession à la propriété de l'entreprise (partage du patrimoine). Dans les deux cas, les étapes peuvent être identifiées et elles peuvent se suivre selon un ordre chronologique assez précis.

Sur le plan de la succession managériale, on distingue quatre étapes principales : l'amorce, le choix, le règne conjoint et le retrait définitif. Avec l'amorce, 
c'est la transmission des valeurs, de la vision et du savoir être et savoir-faire du prédécesseur au successeur. Le choix du successeur se fait habituellement selon des critères comme la confiance, la capacité, la formation, l'expérience, l'intérêt mais aussi selon le rang dans la famille, le sexe et l'implication dans l'entreprise. La phase du règne conjoint débute souvent au moment où le successeur s'implique à plein temps dans l'entreprise et assume de plus en plus de responsabilités managériales. Le prédécesseur quant à lui tend habituellement à se réserver les décisions stratégiques. Vient ensuite le temps de retrait définitif du prédécesseur, soit pour des raisons d'âge, de maladie ou de choix personnel.

Du côté de la transmission de la propriété, le processus est souvent plus court mais implique habituellement un plus grand nombre d'intervenants externes à la famille et à l'entreprise. On peut ramener à trois étapes majeures, le déroulement de ce processus. Il y a d'abord une mise en place des procédures, initiée le plus souvent par le(s) successeur(s). Par la suite, une décision est prise quant au partage du patrimoine, soit à parts égales, au mérite ou encore selon l'implication des individus dans l'entreprise. Enfin, on consulte plusieurs spécialistes (avocat, notaire, comptable, fiscaliste, assureur) et on finalise les actes juridiques.

\section{Recherches et études connexes}

Un certain nombre d'études portant sur la succession dans les entreprises familiales, petites et moyennes, ont été effectuées au cours des dernières années; cependant, la plupart de ces études ont un caractère exploratoire et préliminaire et ont porté sur quelques aspects seulement de la succession. Aucune étude globale n'a vraiment été conduite sur le sujet. Une revue systématique de la littérature portant sur l'entreprise familiale, petite et grande, nous indique par ailleurs, un certain nombre d'éléments et de pistes à prendre en considération lorsqu'on aborde cette problématique; on peut habituellement y retrouver les thèmes suivants :

- La continuité

- La planification

- Le choix des successeurs

- Les problèmes particuliers

- Les mécanismes de transmission

\subsection{La continuite}

Le problème de la continuité dans la direction de la PME familiale peut être envisagé selon deux perspectives principales. Il y a d'abord celle du chef d'entreprise qui doit s'adapter à son rôle au fur et à mesure que son entreprise se développe et se transforme. L'intérêt ici porte principalement sur les transitions que doit effectuer un 
dirigeant de PME lorsque son entreprise passe d'un stade de développement à un autre. L'autre perspective, celle qui nous intéresse particulièrement dans cet article, porte sur le destin de l'entreprise lorsque le dirigeant principal se retire ou décède. Ainsi, dans une recherche (Hugron, 1983) auprès de 35 entreprises familiales du Québec, on note que la passation des pouvoirs de direction représente pour l'entreprise une période critique et potentiellement fatale.

Trois volumes américains récents traitent spécifiquement de cette problématique; bien que ne portant pas seulement sur les PME, cesétudes démontrent avec beaucoup d'à-propos la similitude des difficultés qui assaillent les entreprises familiales aux Etats-Unis et au Canada. Par exemple, après avoir décrit les différents stades d'évolution de l'entreprise familiale, Dyer (1986) montre que c'est la culture prédominante de ce type d'entreprises qui, en fin de compte, déterminera leur habileté à survivre. Pour sa part, Ward (1985) va plus loin et suggère une série d'actions à prendre pour assurer la survie des entreprises familiales aux Etats-Unis dont à peine un tiers se rendent à la deuxième génération. Les auteurs de la troisième étude (Rosenblatt, de Mik, Anderson et Johnson, 1985), mettent surtout l'accent sur les nombreuses tensions, divergences et différences qui surgissent entre les membres des familles impliquées dans des entreprises familiales; on fait état en particulier de la situation délicate des épouses et des filles dans ce genre d'entreprises.

\subsection{La planification de la relève}

Il semble bien qu'une des causes majeures des problèmes de succession et de relève soit l'absence de planification des dirigeants. La plupart des études portant directement ou indirectement sur le sujet mentionne explicitement un manque de planification. Par exemple, Dell'Aniello et Coupal (1977) indiquent que le problème de la succession est essentiellement la conséquence d'un manque de planification et qu'il se présente souvent sous la forme d'une crise parce que personne ne s'en soucie avant qu'il ne devienne crucial.

Aux Etats-Unis, Gomolka (1984) a étudié 223 petites et moyennes entreprises, propriétés de groupes minoritaires et a démontré de façon non-équivoque le manque flagrant de planification successorale; il a été aussi à même d'observer les effets parfois néfastes de cet état de chose. La même constatation a été rapportée par Franklin, Fulmer et Goodwin (1981) auprès de 249 petites et moyennes firmes manufacturières; leurs résultats montrent une grande préoccupation des dirigeants vis-à-vis de leur succession mais peu de planification effectuée, du moins, pas de planification formelle et systématique.

\subsection{Le cholx des successeurs}

Un aspect crucial du processus de remplacement du dirigeant de PME est certes le choix du ou des successeur(s). Ce problème est grandement plus compliqué dans les cas où il se présente plusieurs successeurs potentiels dans la ou les famille(s) 
comme l'ont bien souligné Barnes et Hershon (1976). Ils ont même affirmé que ce choix est tellement difficile pour les dirigeants qu'ils renoncent à leurs responsabilités reportant la décision au dernier moment sans planification, ni préparation du successeur.

Plusieurs raisons ont été avancées pour expliquer ces réactions. Par exemple, Beckhard et Dyer (1983) prétendent qu'effectuer un changement de cette nature est particulièrement compliqué dans une entreprise familiale à cause de plusieurs facteurs, dont la résistance du dirigeant. Ce type de décisionn'est pas facile parce que plusieurs éléments sociaux, psychologiques, familiaux et d'affaires entrent en considération; c'est souvent une question de confiance envers le successeur qui est prédominante dans ce choix.

Il y a cependant une dimension sur laquelle toutes les études convergent, c'est le choix du fils âné qui est, de loin le plus souvent, le successeur dans l'entreprise familiale. On perçoit ce choix comme naturel et comme faisant partie des responsabilités dévolues au fils aîné. Historiquement, il semble que les filles aient été déchargées de cette responsabilité particulière; selon une étude de Business Week (1982), seulement 500 femmes étaient aux commandes d'une entreprise familiale en 1982 aux Etats-Unis.

\subsection{Les problèmes particuliers}

Parmi les problèmes les plus souvent rencontrés dans les successions d'entreprises familiales, il y a celui du manque d'intérêt des enfants pour assumer la relève. Selon une étude d'Ambrose (1983), les dirigeants plus âgés déclarent majoritairement que leurs enfants ne sont pas intéressés à l'entreprise familiale. En réalité, c'est souvent parce que les dirigeants ont attendu trop longtemps avant d'intéresser leurs enfants à l'entreprise et ces demiers ont passé l'âge idéal pour prendre la succession. Lorsqu'on leur offre la relève, les enfants sont déjà souvent bien établis dans leur propre carrière et leurs intérêts du moment ne coïncident pas avec ceux exigés pour la direction d'une entreprise. Encore une fois, cette situation est souvent la conséquence d'une absence de planification et de préparation à la relève. Par ailleurs, les enfants ont tendance à percevoir l'entreprise familiale comme une option parmi plusieurs. Selon la même étude, les enfants ne verraient pas nécessairement dans l'entreprise familiale, un lieu de grande satisfaction personnelle, certainement moins que leurs parents. Il découle de ces constatations que pour augmenter l'intérêt des enfants vis-à-vis de l'entreprise familiale et accroître leur prédisposition à la succession, il s'agit de les intégrer le plus tôt possible dans l'entreprise et leur faire assumer des responsabilités de direction.

Cependant, lors de l'arrivée du ou des successeur(s) dans l'entreprise, un certain jeu de pouvoir se produit qui entraîne des ajustements, des frictions, voire même des affrontements. Habituellement, le propriétaire-dirigeant ne cédera sa place que s'il est convaincu que le successeur pourra éventuellement faire aussi bien que lui. Des problèmes pourront aussi se poser avec les employés. L'arrivée d'une 
nouvelle administration et le retrait du propriétaire-dirigeant peut amener certains changements dans la culture de l'entreprise; il y a souvent à revoir les attentes mutuelles et à rebâtir le climat de confiance. Une succession graduelle sur plusieurs années peut parer grandement ce genre de difficultés.

\subsection{Les mécanismes de transmission}

Probablement le meilleur mécanisme de transmission est encore une bonne planification et préparation. A cet effet, Longenecker and Schoen (1978) proposent un modèle de succession en sept étapes dont trois se situent avant l'entrée dans l'entreprise du successeur éventuel. Peu importe le mécanisme utilisé, il faut évidemment que les parties y voient leurs intérêts et avantages qui souvent sont mieux mis en évidence par un tiers, professionnel et conseiller. L'aide extérieure doit être recherchée non seulement pour conseiller le propriétaire-dirigeant mais aussi le successeur et la famille.

La plupart des études sur la succession ont privilégié le point de vue du propriétaire et de son entreprise mais comme le souligne Birley (1986) rarement celui du successeur et de la famille. On lui attribue parfois à tort les raisons de l'insuccès d'une transmission de direction et de propriété; les rôles, les attenteset les motivations de toutes les parties prenantes à la transaction doivent être pris en considération.

\section{Questions de recherche}

Les attentes spécifiques découlant de cette recherche sont principalement basées sur les perceptions des individus qui détiennent actuellement le contrôle des entreprises. Les auteurs ont tenté de savoir comment, par exemple, réagissent les propriétaires-dirigeants québécois face à la possibilité de confier un jour leur entreprise à leur conjoint, ou à leur fille. Comment voient-ils cette éventualité dans le cas de décès ou de maladie prolongée. En fait, ils ont cerné les questions se rapportant à la relève des propriétaires-dirigeants (de leur vivant) et à leur succession (après une absence subite, par exemple).

La diversité du monde entrepreneurial propose différents angles derecherche en vue de mieux cerner ces perceptions : pourraient-elles par exemple, varier selon les secteurs (industriel, commercial ou services), les formes juridiques (les statuts de propriétaire et d'actionnaire ont été considérés), l'âge et la taille de la PME, l'état de la planification personnelle successorale, l'intégration de l'épouse ou des enfants dans les activités de l'entreprise, la composition de la famille (enfants, groupe d'âge, rang), l'âge du dirigeant, etc.? Il sera démontré ultérieurement que des différences sensibles informent l'hypothèse voulant qu'il n'y ait qu'une seule perception globale et uniforme de la relève et de la succession féminine. 
Les auteurs ont voulu prendre une mesure du caractère familial de certaines entreprises. Il ne s'agit pas de comprendre les concepts de relève et de succession dans leur forme culturelle ou générale : l'approche est plus spécifique, basée sur l'aspect familial de la vie des propriétaires-dirigeants. En d'autres termes, l'étude discerne ce qui reste de «familial» dans le contrôle des entreprises afin d'établir les liens appropriés entre l'aspect «familial» et l'aspect «entreprise».

\section{$4 \quad$ Méthodologie}

\subsection{Plan d'échantillonnage}

La population visée comprenait, àl'origine, 1388 entreprises. L'échantillon final contient 549 questionnaires, complétés et retournés par le courrier. Le taux de réponse est de l'ordre de $39,5 \%$, ce qui est considéré comme très élevé pour ce genre d'étude.

\subsection{Description de l'échantillon}

Le secteur primaire n'est pas présent dans le plan d'échantillonnage. Les autres principaux secteurs économiques sont proportionnellement bien représentés dans l'étude, tel que démontré dans le tableau 1.

Tab. 1. Secteurs d'activités des répondants

\begin{tabular}{|l|c|c|}
\hline Secteur & N & $\%$ \\
\hline Manufacturier & 163 & 29,9 \\
Commerce de détail & 157 & 28,8 \\
Services & 124 & 22,7 \\
Commerce de gros & 101 & 18,5 \\
\hline TOTAL & 545 & 100,0 \\
\hline
\end{tabular}

L'échantillon est fortement masculin $(94,6 \%)$. La moyenne d'âge des répondants est 47,24 ans. Une proportion importante des répondants sont mariés $(88,2 \%)$. Le nombre d'enfants par famille se situe autour de 2,7 . La famille moyenne compte 1,4 fils et 1,3 fille.

En ce qui concerne l'âge des enfants, la classe modale chez les fils se situe dans le groupe des $0-17$ ans $(38,4 \%)$. Cette constatation signifie que près des deux 
cinquièmes du nombre total des fils ne sont pas, au moment de l'enquête, en âge «légal» de succéder ou prendre la relève (c'est-à-dire qu'ils sont mineurs). Quant aux filles, légèrement moins nombreuses, le statut de mineur touche $36,2 \%$ d'entre elles.

Le type de propriété se distribue de la manière suivante : $33 \%$ des répondants sont propriétaires de leur entreprise; $60 \%$ sont actionnaires majoritaires; $6,6 \%$ sont actionnaires minoritaires.

La taille de l'entreprise est définie ici en fonction du nombre d'employés. Ainsi, la «petite» PME compte entre 3 et 15 employés et représente $28 \%$ de l'échantillon; la «moyenne» $P M E$ comprend entre 16 et 50 employés et constitue près de la moitié des répondants $(50,8 \%)$; la «grande» PME se compose de 51 employés et plus et représente $21,2 \%$ de l'échantillon.

L'âge moyen des entreprises étudiées est de 23 ans. Le tableau 2 présente ces entreprises regroupées sous quatre catégories, selon leur âge.

Tab. 2. Catégorie d'entreprises selon leur áge depuis leur fondation

\begin{tabular}{|ll|r|r|}
\hline Etape & Limites & N & $\%$ \\
\hline Démarrage & Entre 1 et 4 ans & 20 & 3,7 \\
Croissance & Entre 5 et 14 ans & 177 & 32,5 \\
Maturité 1 & Entre 15 et 29 ans & 189 & 34,7 \\
Maturité 2 & Entre 30 et 134 ans & 158 & 29,0 \\
\hline TOTAL & & 544 & 100,0 \\
\hline
\end{tabular}

\section{$5 \quad$ Modèle}

Trois types de variables ont été étudiées. Les variables indépendantes sont par exemple l'état civil du répondant, son âge, la taille de l'entreprise, etc. Les variables déterminantes sont l'importance de la relève ou de la succession, l'état de la planification, l'aptitude (réfere à la personne la plus apte, selon le dirigeant, à prendre sa relève) et la succession en cas d'absence subite. D'autres variables déterminantes ont été aussi considérées : l'ordre d'importance des candidatures à la relève, l'intégration des personnes aux activités de l'entreprise et la perception des cadres et des employés quant à la présence du fils, de la fille et du conjoint auprès d'eux. Notons que ces trois dernières variables servent surtout à expliquer certaines conditions qui pourront influencer les autres variables déterminantes; on les appelle 
co-déterminantes. Enfin, les variables dépendantes sont représentées dans cette étude (Figure 1) par les perceptions des dirigeants envers plusieurs facettes de la relève et de la succession dans leur entreprise.

Fig. 1. Modèle perceptuel de la relève et succession

\begin{tabular}{|c|c|c|}
\hline $\begin{array}{l}\text { Variables } \\
\text { Indépendantes }\end{array}$ & $\begin{array}{l}\text { Variables } \\
\text { déterminantes }\end{array}$ & $\begin{array}{l}\text { Variables } \\
\text { dépendantes }\end{array}$ \\
\hline \multicolumn{3}{|l|}{ Etat civil } \\
\hline Nombre de fils & Importance de la relève & Perception \\
\hline Nombre de filles & Planification & \\
\hline Age des enfants & Transmission (type) & \\
\hline Type de propriété & Aptitude à la relève & Perception \\
\hline Taille de l'entreprise & Circonstances & \\
\hline Age de l'entreprise & Rang du candidat & \\
\hline Secteur d'activité & Intégration dans l'entreprise & Perception \\
\hline Sexe du répondant & Perception des employés & \\
\hline \multicolumn{3}{|l|}{ Age du répondant } \\
\hline \multicolumn{3}{|l|}{ Temps de possession } \\
\hline Causes possibles & Contingences & Perceptions \\
\hline
\end{tabular}

\section{Résultats}

\subsection{Importance de la relève et de la succession}

La grande majorité des dirigeants d'entreprises considèrent la relève et la succession comme étant soit très important ou important $(79,6 \%)$.

Les répondants provenant du secteur du commerce de gros sont ceux qui considèrent le plus la relève et la succession comme étant très important $(63,3 \%)$. Cette importance ne se traduit pas de la même façon selon la taille de la PME. Ainsi, les dirigeants de la grande PME $(53,4 \%)$ sont plus préoccupés par la relève et la succession. Les dirigeants de la petite entreprise considèrent la question comme étant peu importante dans une plus forte proportion (20,8\%).

Plus le dirigeant est âgé, plus le sujet de la relève et de la succession devient important. Près du tiers des répondants âgés de 40 ans et moins considèrent cet aspect peu important ou n'y songent même pas. Cette relation entre les deux variables 
semble normale; les dirigeants les plus âgés (87\%) étant plus près de leur retraite, la relève devient plus pressante.

Les répondants qui n'ont pas de fils considèrent la relève et la succession de façon moins importante (19\% croient que le sujet est peu important; $13,8 \%$ n'y songent pas). L'augmentation du nombre de fils accroît l'importance accordée au sujet; la majorité des dirigeants $(54,6 \%)$ qui ont deux fils et plus le considère très important. En ce qui concerne la relation avec le nombre de filles, les tendances sont assez semblables. De manière générale, l'importance accordée à la relève et à la succession augmente en fonction du nombre d'enfants. Par ailleurs, le groupe des répondants âgés de 55 ans et plus, qui ont des enfants en âge de leur succéder, est le plus préoccupé par la question.

Lorsque les enfants sont intégrés à la vie de l'entreprise (ou ont déjà été intégrés), les dirigeants indiquent majoritairement (62,9\% dans le cas du fils) que la question est d'une grande importance.

En résumé, le portrait type du dirigeant qui considère la relève et la succession comme étant très important est un homme âgé de 50 ans et plus, oeuvrant dans le commerce de gros ou le secteur manufacturier, ayant plusieurs enfants (fils et filles) en âge de lui succéder et intégrés à la vie de l'entreprise - au point de les considérer au premier rang au niveau de la relève.

\subsection{Planification de la relève et de la succession}

Plus du tiers des dirigeants (35\%) ont officialisé leur décision quant à la planification de la succession de leur entreprise, mais près de $30 \%$ des répondants ont mentionné avoir une approche plutôt informelle. De plus, $20,5 \%$ des décisions prises par les dirigeants reposent sur des «contrats psychologiques» entre gens concernés.

Les entreprises commerciales «officialisent» (la succession est inscrite dans un document officiel) davantage leur décision quant à leur succession. Le secteur manufacturier démontre le taux d'officialisation le plus faible (27,2\%); par contre, dans ce même secteur on avise quand même son entourage de sa décision $(32,7 \%)$. Les dirigeants qui n'ont pas planifié leur relève et leur succession sont surtout présents dans le domaine des services (27,9\%).

Les grandes et moyennes PME sont celles qui officialisent le plus leur décision quant à la succession (respectivement $39,7 \%$ et $37,2 \%$ ). La petite PME semble la moins systématique en ce qui concerne la planification $(21,5 \%$ des répondants se situent au niveau de l'intention; $23,6 \%$ n'ont pas planifié).

En résumé, on peut dresser le portrait type du dirigeant qui officialise sa décision quant à sa relève et à sa succession. Celui-ci a un nombre d'enfants plus élevé que la moyenne (1,6 fils et 1,4 fille); il a atteint la cinquantaine; il est à la tête d'une entreprise qui a près d'un quart de siècle et qui génère un chiffre d'affaires élevé (plus de 8 millions de \$); il est surtout propriétaire d'une grande PME qui se situe dans le secteur commercial (gros et détail). Egalement, il a des enfants en âge de lui 
succéder. Ceux-ci sont intégrés aux activités de l'entreprise (43,6\% dans le cas du fils; $37,9 \%$ dans le cas de la fille). Il choisit son fils ou sa fille au premier rang en ce qui a trait à sa relève, mais surtout son fils s'il le considère apte $(44,4 \%$ dans le cas du fils; $32,7 \%$ dans le cas de la fille). Enfin, il privilégie les professionnels (49\%) lorsqu'il a besoin de conseils à ce sujet et prévoit de prendre sa retraite vers 58 ans.

\subsection{Aptitude à la relève}

Selon les dirigeants, les personnes les plus aptes à prendre leur relève sont, par ordre d'importance : le fils $(39,7 \%)$, l'associé $(20,6 \%)$, une personne sans lien de parenté $(16,9 \%)$, la fille $(9,6 \%)$, l'épouse $(7,9 \%)$ et le frère $(4,2 \%)$. La majorité de l'échantillon $(57,2 \%)$ a donc une perception «familiale» de l'aptitude à la relève.

Ce sont les dirigeants du secteur commercial (gros et détail) qui considèrent le plus l'aptitude de leurs enfants à la relève; ce secteur est le plus «familial». Les dirigeants du secteur manufacturier se tournent davantage vers lescapacités extérieures à la famille immédiate : la majorité d'entre eux (55\%) considèrent les «autres» (associés, frères, parents et amis - les trois derniers groupes sont marginalement représentés), comme étant les plus aptes à la relève. La conjointe et la fille sont faiblement reconnus quant à l'aptitude à la relève, et ce dans tous les secteurs.

L'aptitude du fils est surtout reconnue dans la moyenne PME $(45,6 \%)$. C'est au niveau de la petite PME que le fils reçoit le moins de reconnaissance $(31,6 \%)$. Par ailleurs, plus l'entreprise prend de l'ampleur, plus le dirigeant se tourne vers les «autres» pour assurer la relève (jusqu'à 50\% dans le cas de la grande PME).

L'aptitude du fils est surtout reconnue par le dirigeant qui est actionnaire majoritaire $(41,5 \%)$. Si le fils est intégré à la vie de l'entreprise, c'est dans une forte proportion $(70,9 \%)$ qu'il sera considéré comme étant le plus apte à la relève. Presque la moitié des dirigeants $(49,2 \%)$ ayant confiance à l'aptitude de leur fils ont officialisé leur décision.

Le portrait type du dirigeant qui choisit son fils comme ayant le plus d'aptitude à la relève a en moyenne 3,5 enfants (la moyenne générale de l'échantillon est de 2,7); il a atteint la cinquantaine et prévoit de prendre sa retraite à 58 ans; l'entreprise qu'il dirige est en opération depuis près de 28 ans et son chiffre d'affaires dépasse 6 millions de $\$$ tandis que le nombre d'employés de l'entreprise atteint le chiffre de 43.

\subsection{La relève ou la succession en cas d'absence subite}

L'aptitude à la relève réfere plutôt à une situation à long terme et aux aspirations des dirigeants. La relève ou la succession en situation d'urgence indique la perception des dirigeants dans une perspective à court terme. Elle implique une situation plus près de la réalité vécue par l'entreprise et met en perspective la compétence des personnes appelées à en prendre la direction. A court terme, les 
dirigeants semblent conditionnés par les rapports quotidiens qu'ils entretiennent avec leurs associés et l'aptitude de ceux-ci à prendre la relève immédiatement. Ainsi, ils font davantage confiance à des personnes extérieures à la cellule familiale $(58,3 \%)$ qu'à leur propre famille $(41,7 \%)$.

Le secteurmanufacturier demeure le moins «familial»; ainsi dans la catégorie «autres» qui regroupait 55\% des choix quant à l'aptitude à la relève, $72,6 \%$ passent à ces choix lorsqu'il s'agit d'absence subite. Le secteur commercial (gros) est le seul qui demeure majoritairement familial.

De plus, la catégorie «autres» est mentionnée par une forte proportion des répondants $(72,1 \%)$ au niveau de la grande PME.

Ainsi, on peut dégager certaines comparaisons entre les répondants en ce qui a trait au choix de la personne qui prendrait la relève en cas d'absence subite. Par exemple, le répondant à profil familial est en moyenne plus vieux que celui à profil extra-familial (56,6 ans vs 44,2 ans); son entreprise est plus âgée (32,5 ans vs 21,6 ans) et sa famille est plus nombreuse. La fille et le conjoint sont encore une fois choisis surtout dans les petites PME, tandis que le fils est prioritaire dans les moyennes PME.

\subsection{La perception de la relève familiale par l'entourage de l'entreprise}

Presque la moitié des répondants croient que leurs enfants seraient bien perçus par leur entourage de travail, si l'un d'entre eux prenait effectivement la relève; la fille obtient un taux perceptuel positif de $48,3 \%$ alors que le fils enregistre 49,5\%. Par contre, le conjoint se voit assigner une perception d'ensemble plutôt négative (la perception négative qui lui est attribuée est la plus élevée des trois catégories).

\section{Conclusion}

La perception de la relève et de la succession par le dirigeant de PME est fondée sur deux principales constatations : la situation actuelle de son entreprise (avec les besoins d'une direction à court terme qui font appel à la compétence des personnes déjà en place) et ses aspirations personnelles (sur la propriété à long terme du patrimoine économique par le successeur de son choix - souvent «désiré»).

La perception du dirigeant quant à sa relève et à sa succession varie selon les secteurs économiques, les formes juridiques, la taille et l'âge de l'entreprise, l'âge du dirigeant, la composition de sa famille et l'intégration dans l'entreprise de ses enfants ou de son conjoint. Mais l'aspect «familial» persiste à travers l'ensemble des perceptions. Par contre, il y a l'aspect «entreprise» qui intervient lorsque les besoins 
et les intérêts de celle-ci ne peuvent fournir d'ancrage aux aspirations familiales de son dirigeant. Les choix de ce dernier se tournent alors vers l'extérieur de sa famille.

Les dirigeants du secteur commercial reconnaissaient davantage leurs enfants comme étant aptes à la relève : le fils est surtout mentionné au niveau du commerce de gros et la fille dans le commerce de détail (à l'intérieur d'entreprises relativement plus petites, en nombre d'emplois et en chiffre d'affaires).

Du côté de la transmission de la propriété, le processus est souvent plus court mais implique habituellement un plus grand nombre d'intervenants externes à la famille et à l'entreprise. On peut ramener à trois étapes majeures, le déroulement de ce processus. Il y a d'abord une mise en place des procédures, initiée le plus souvent par le(s) successeur(s). Par la suite, une décision est prise quant au partage du patrimoine, soit à parts égales, au mérite ou encore selon l'implication des individus dansl'entreprise.Enfin, on consulte plusieurs spécialistes (avocat, notaire, comptable, fiscaliste, assureur) et on finalise les actes juridiques.

La perception du dirigeant en ce qui concerne la possibilité de voir un jour sa fille prendre la relève ou lui succéder est surtout fondée sur des aspirations familiales : à défaut de fils apte ou disponible, le dirigeant tourné vers sa famille étendra le concept «enfants» pour y inclure sa fille... Mais en réalité, la place effectivement accordée à la fille semble encore marginale.

L'intégration est un facteur important pour la promotion de la fille. Contrairement au fils (qui est promu à la tête de l'entreprise à la fois selon les aspirations et la réalité), celle-ci doit être intégrée aux activités de l'entreprise afin d'être pleinement reconnue par le dirigeant. Autrement dit, elle doit démontrer son intérêt et ses capacités avant d'être considérée comme une candidate valable (chez le fils, la considération vient avant la démonstration!).

Enfin, les résultats de cette recherche font ressortir que les processus de transmission peuvent débuter assez tôt dans la vie d'une entreprise et se poursuivent à plusieurs niveaux en faisant appel à des intervenants possédant des intérêts et des expertises variés. Pour les dirigeants d'entreprises, le message véhiculé par ces études peut se résumer à trois principes de base :

- la succession et la relève doivent se préparer assez tôt et se planifier;

- ces processus, comme d'autres éléments de la gestion d'une entreprise, peuvent se gérer et faire partie intégrante de la stratégie des dirigeants;

- puisqu'il s'agit de questions complexes, on doit faire appel aux gens de l'entourage immédiat et de l'extérieur pour faciliter les transitions.

Il reste cependant à souhaiter que les dirigeants d'entreprise prennent conscience de façon pratique de la dimension relève et succession de leur entreprise pour le mieux-être de la famille et de la collectivité. 


\section{BIBLIOGRAPHIE}

AMBROSE, D.M., Transfer of the family-owned business, Journal of Small Business Management, vol. 21, $\mathrm{n}^{\circ} 1,1983$, janvier, pp. 49-56.

BARNES, L.B. et HERSHON, S.A., Transferring power in the family business, Harvard Business Review, 1976, juillet/août.

BECKHARD, R. et DYER, W.G., Managing change in the family firm : Issues and strategies, Sloan Management Review, 1983, printemps.

BIRLEY, S., Succession in the family firms : the inheritor's view, Journal of Small Business Management, vol. 24, no 3, 1986, juillet, p. 36-43.

BUSINESS WEEK, When a daughter takes over the family business, 1982, 29 mars.

DELL'ANIELLO, P., et COUPAL, M., Cahier de recommandations portant sur les petites et moyennes entreprises québécoises, Québec : Ministère de l'Industrie et du Commerce, novembre, 1977,48 pages.

DYER, G.W., Cultural Change in Family Firms, San Francisco, Jossey-Bass, 1986.

FRANKLIN, S.G., FULMER, R.M. et GOODWIN, J.S., Analyzing succession strategies for small firms, Proceedings, International Council for Small Business, 1981, pp. 18-24.

GASSE, Y., Entrepreneurial Characteristics and Practices : A study of the dynamics of small business organizations and their effectiveness in different environments, Monographie de recherche, M.I.C., Ottawa, 1977.

GOMOLKA, E.G.,Entrepreneurial succession in minority-owned businesses : An empirical analysis, Proceedings, International Council for Small business, 1984, pp. 55-62.

HUGRON, P., La succession dans les entreprises familiales au Québec, Montréal, Ecole des Hautes Etudes Commerciales, rapport de recherche $n^{\circ} 83-22,1983,65$ pages.

HUGRON, P., Succession managériale dans les PME familiales au Québec, Rapport de recherche intérimaire, Institut de Recherches Politiques, Programme sur la PME, 1985.

LONGENECKER, J.G. et SCHOEN, J.E., Management succession in the family business, Journal of Small Business Management, 1978, juillet.

ROSENBLATT, P.C., de MIK, L., ANDERSON, P.M. et JOHNSON, P.A., The Family in Business, San Francisco, Jossey-Bass, 1985.

WARD, J.L.,Keeping the Family Business Healthy, San Francisco, Jossey-Bass, 1985. 